\title{
IMAGINARY POWERS OF THE DEDEKIND ETA FUNCTION
}

\author{
BERNHARD HEIM, MARKUS NEUHAUSER, AND FLORIAN RUPP
}

\begin{abstract}
In this paper complex powers of the Dedekind eta function are studied. The vanishing of the $n$-th Fourier coefficients are labeled by the roots of an attached polynomial $p_{n}(x)$. We study these polynomials, and their values and roots distribution. The considered polynomials of degree $n \leq 700$ are verified as Hurwitz polynomials. We study the value distribution of the polynomials restricted to the imaginary axis.
\end{abstract}

\section{INTRODUCTION}

The main purpose of this paper is to study the distribution of the roots of a family of recursively defined polynomials $p_{n}(x)$. The values on the imaginary axis it are studied. The roots label powers of the Dedekind eta function $\eta$, whose $n$-th Fourier coefficient is vanishing.

Let $\sigma(n):=\sum_{d \mid n} d$ and let $p_{0}(x):=1$, then

$$
p_{n}(x):=\frac{x}{n} \sum_{k=1}^{n} \sigma(k) p_{n-k}(x) .
$$

The root distribution is closely related to the works of Euler, Jacobi, Lehmer, Serre, and Gordon and Ono on lacunary and super lacunary eta products and their relations to theta series ([Se81, Se85, On98, On03, Ko11]).

In his celebrated paper [Se85], Serre proved that even powers $\eta(\tau)^{r}$ of the Dedekind eta function

$$
\eta(\tau):=q^{\frac{1}{24}} \prod_{n=1}^{\infty}\left(1-q^{n}\right), \quad\left(q:=e^{2 \pi i \tau}, \operatorname{Im}(\tau)>0\right)
$$

are lacunary if and only if the powers are $2,4,6,8,10,14$ or 26 . Based on results of Ribet [Ri77], Serre actually proved that for $f(\tau):=\eta(12 \tau)^{2}$,

$$
f^{k}(\tau)=q^{k} \sum_{n=0}^{\infty} \widetilde{p_{r}}(n) q^{12 n} \quad(r=2 k)
$$

has complex multiplication if and only if $k=1,2,3,4,5,7,13$ (see also [Se81]). The proof depends on vanishing properties of forms with respect to Hecke operators $T_{p}$, where $p \equiv-1(\bmod 12)$. Finally the property that for $0 \leq m \leq 10, p=11$ and

2010 Mathematics Subject Classification. 26C10, 11F20; Secondary 11F30, 11F37, 12 D10.

Key words and phrases. Fourier Coefficients, Euler Products, integer-valued polynomials, Lehmer Conjecture, Roots of Polynomials. 
$k+m \equiv 0(\bmod 11):$

$$
\widetilde{p_{2 k}}(m) \neq 0 \text { for } k \neq 2,3,4,5,7,13
$$

has to be explicitly checked. Therefore the integral roots of $\widetilde{p_{r}}(m)$ as a polynomial in $r$ up to degree $m=10$ are studied. These polynomials and their factorization are obtained from the pioneering work of Newman [Ne55], see also [Se85], section 1.2.

$$
\begin{aligned}
\widetilde{p_{r}}(0) & =1 ; \widetilde{p_{r}}(1)=-r ; \widetilde{p_{r}}(2)=r(r-3) / 2 ; \widetilde{p_{r}}(3)=-r(r-1)(r-8) / 3 ! ; \\
\widetilde{p}_{r}(4) & =r(r-1)(r-3)(r-14) / 4 ! ; \widetilde{p_{r}}(5)=-r(r-3)(r-6)\left(r^{2}-21 r+8\right) / 5 ! ; \\
\widetilde{p_{r}}(6) & =r(r-1)(r-10)\left(r^{3}-34 r^{2}+181 r-144\right) / 6 ! ; \\
\widetilde{p_{r}}(7) & =-r(r-2)(r-3)(r-8)\left(r^{3}-50 r^{2}+529 r-120\right) / 7 ! ; \\
\widetilde{p_{r}}(8) & =r(r-1)(r-3)(r-6)\left(r^{4}-74 r^{3}+1571 r^{2}-9994 r+4200\right) / 8 ! ; \\
\widetilde{p_{r}}(9) & =-r(r-1)(r-3)(r-4)(r-14)(r-26)\left(r^{3}-60 r^{2}+491 r-120\right) / 9 ! \\
\widetilde{p_{r}}(10) & =r(r-1) P(r) / 10 !
\end{aligned}
$$

The polynomial $P(r)$ has integral coefficients and is irreducible over $\mathbb{Q}$.

$$
\begin{aligned}
P(r)= & r^{8}-134 r^{7}+6496 r^{6}-147854 r^{5}+1709659 r^{4} \\
& -10035116 r^{3}+28014804 r^{2}-29758896 r+6531840 .
\end{aligned}
$$

The recursively defined polynomials $p_{n}(x)$ satisfy the fundamental equation

$$
\sum_{n=0}^{\infty} p_{n}(z) q^{n}=\prod_{n=1}^{\infty}\left(1-q^{n}\right)^{-z}
$$

(see also Apostol [Ap76], chapter 14). Hence $p_{m}(r)=\widetilde{p_{r}}(m)$.

In this paper, the polynomials and the distribution of roots are studied systematically and on an experimental level apparently for the first time. It follows directly from the definition (1.1) and the equivalent identity (1.5) that the $p_{n}(x)$ are integervalued polynomials with decomposition

$$
p_{n}(x)=\frac{x}{n !} p_{n}^{*}(x) .
$$

Here $p_{n}^{*}(x) \in \mathbb{Z}[x]$, normalized and of degree $n-1$, and all coefficients are positive. For example:

$$
p_{1}^{*}(x)=1, p_{2}^{*}(x)=x+3, p_{3}^{*}(x)=x^{2}+9 x+8 .
$$

Note, since $p_{2}^{*}(-3)=0$, we know that the second coefficient of $\eta(\tau)^{3}$ is root. Actually it is the only second coefficient of any non-trivial power of $\eta(\tau)$. Due to Euler, the root -1 appears infinitely many times

$$
\prod_{n=1}^{\infty}\left(1-q^{n}\right)=\sum_{n=-\infty}^{\infty}(-1)^{n} q^{n(3 n-1) / 2} .
$$

The Lehmer conjecture [Le47] states that there exist no $n \in \mathbb{N}$, such that -24 is zero, or equivalent $x+24$ never divides $p_{n}^{*}(x)$. 
We start in section 2 to examine the roots of $p_{10}(x)$. The 10 th polynomial is interesting, since it is the first polynomial with non-real roots. In section 3 we calculate the number of integral, irrational, and non-real roots up to $n \leq 50$. We record the integral roots from $n=1$ to 50 . It also seems worth to mention that for $n \leq 700$ (numerically checked) all polynomials $p_{n}^{*}$ are Hurwitz polynomials. In section 4 we prove a result, which connects properties of the roots of $p_{n}(x)$ and $\left|p_{n}(s+i t)\right|$ $(s \in \mathbb{R})$, as a function in $t$. Then in section 5 the values $p_{n}(i t)$ are studied for $n$ small and large. In section 6 the Hurwitz criterion is presented and applied at $p_{n}^{*}$ for $n=1,2,3,4,5$. Finally in the appendix we also record all polynomials $p_{n}^{*}$ and their factorization over $\mathbb{Q}$ for $n=1$ to $n=25$, in order to extend the list of Newman and Serre.

\section{First Complex Roots}

The polynomial $p_{10}(x)$ for $n=10$ is special. Due to Newman and Serre it is known that for $n \leq 4$ the polynomials are completely reducible over $\mathbb{Q}$. Newman and Serre also determined the irreducible factors up to $n=10$. Our analysis shows that for $n=10$ the first time non-real roots appear. There are 2 integral, 6 irrational, and 2 non-real roots. We recall the explicit form of $10 ! p_{10}(x)$ :

$$
\begin{array}{r}
x^{10}+135 x^{9}+6630 x^{8}+154350 x^{7}+1857513 x^{6}+11744775 x^{5} \\
+38049920 x^{4}+57773700 x^{3}+36290736 x^{2}+6531840 x
\end{array}
$$

This polynomial decomposes over $\mathbb{Q}$ into three irreducible factors of degree one, one, and eight.

$$
x(x+1) R(x) .
$$

The degree eight factor is given by

$$
\begin{aligned}
R(x)= & x^{8}+134 x^{7}+6496 x^{6}+147854 x^{5}+1709659 x^{4} \\
& +10035116 x^{3}+28014804 x^{2}+29758896 x+6531840 .
\end{aligned}
$$

Next, we record the values of the roots. 


\begin{tabular}{|c|r|r|l|}
\hline Roots & $\mathbb{Z}$ & $\mathbb{R} \backslash \mathbb{Z}$ & $\mathbb{C} \backslash \mathbb{R}$ \\
\hline$\alpha_{1}$ & 0 & & \\
$\alpha_{2}$ & -1 & & \\
$\alpha_{3}$ & & $\approx-0.292$ & \\
$\alpha_{4}$ & & $\approx-1.710$ & \\
$\alpha_{5}$ & & $\approx-13.055$ & \\
$\alpha_{6}$ & & $\approx-17.674$ & \\
$\alpha_{7}$ & & $\approx-32.165$ & \\
$\alpha_{8}$ & & $\approx-58.180$ & \\
$\alpha_{9}$ & & & $\approx-5.462+0.708 i$ \\
$\alpha_{10}$ & & & $\approx-5.462-0.708 i$ \\
\hline
\end{tabular}

Note, all non-trivial roots have negative real part. This is maybe not just a coincidence. Of course, this has to be checked only for roots in $\mathbb{C} \backslash \mathbb{R}$, since all our polynomials have real and positive coefficients (actually we look at $p_{n}^{*}(x)$ to be precise). Polynomials with such a special property have been studied by Hurwitz [Hu95]. Hurwitz polynomials appear typically as characteristic polynomials of linear systems of differential equations. They control the stability of the underlying system. The problem of testing the stability was first raised in 1868 by Maxwell [Ma68]. See [AL13] for definitions and a short overview.

Definition. Polynomials $p(x) \in \mathbb{R}[x]$ are called Hurwitz polynomials if all their roots have negative real part.

Remark. A numerical study of the roots of $p_{n}(x)$ up to $n=700$ verifies that our first observation remains true. They are all Hurwitz polynomials. 

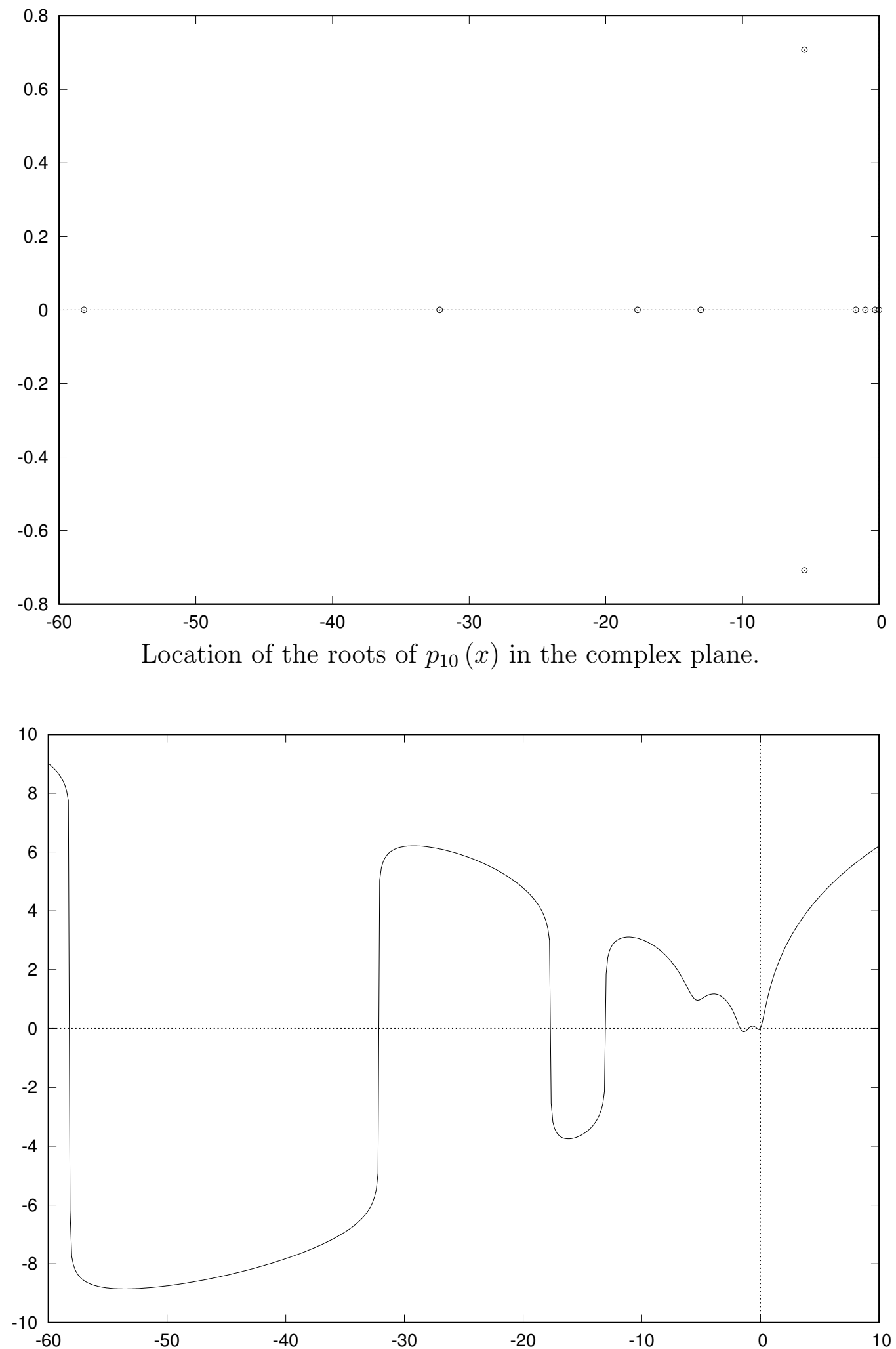

Graph of $p_{10}(x)$ with the transformation of $y \mapsto \operatorname{sgn}(y) \log _{10}(1+|y|)$ on the $y$-values to see the sign changes and have an almost 'logarithmic' scale. 
2.1. Value distribution for $\left|p_{n}\left(\alpha_{9}\right)\right|$. We already know from the formula of Euler that $\alpha_{2}=-1$ is a root of infinitely many polynomials $p_{n}(x)$. In the following we study the chances of $\alpha_{9}$ to have the same property. Therefore, we have displayed the absolute values of $p_{n}\left(\alpha_{9}\right)$ for $n \leq 25$

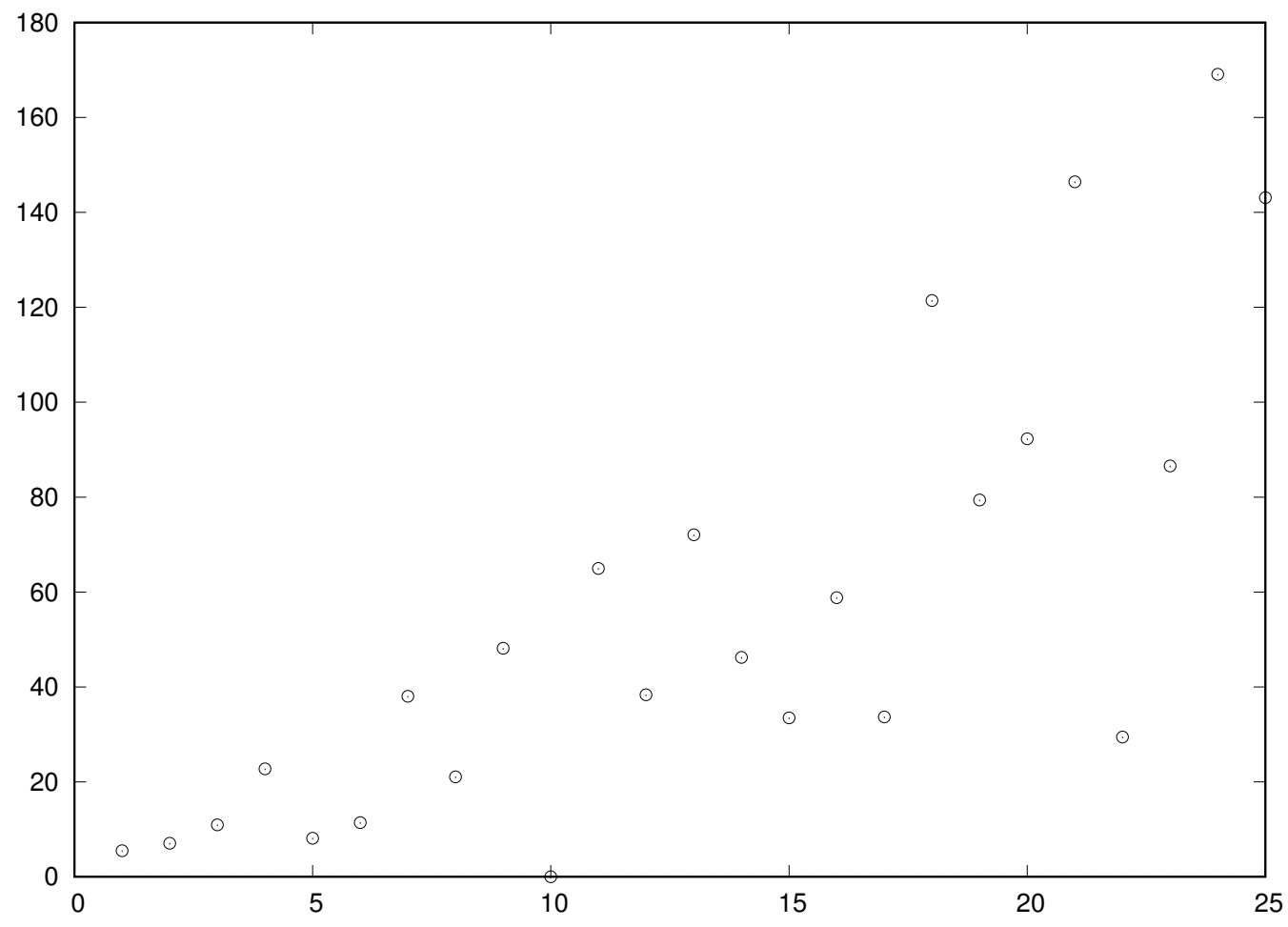

Absolute value of the iteration of the polynomials $p_{n}\left(\alpha_{9}\right)$ for $n=1, \ldots, 25$.

The graph shows some scattering. A more detailed analysis also shows that real and imaginary parts have sign chances. Nevertheless plotting for $n=1, \ldots, 1000$ the complex values strongly indicated that $\alpha_{9}$ is only a root for $n=10$. 


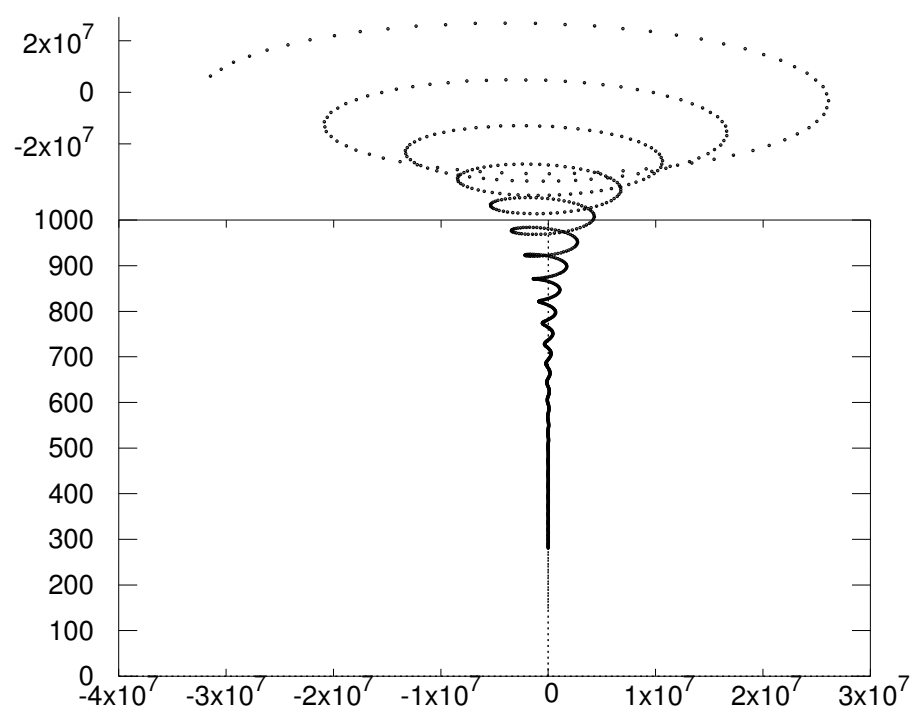

Complex values of the iteration of the polynomials $p_{n}\left(\alpha_{9}\right)$ for $n=1, \ldots, 1000$. The horizontal axis ( $x$-axis) is the real axis and the vertical axis is a projection of the $y$-z-plane where the $y$-coordinate is the iteration step $n=1, \ldots, 1000$ and the $z$-coordinate is the imaginary part.

\section{Distribution of Roots}

The algebraic nature of the roots of $p_{n}(x)$ vary from integral, irrational to nonreal. For $n \leq 4$ all are integral. It seems that this is only true in these cases, as our calculations show. It would be interesting to prove it. Note, the complex roots come in pairs, since our polynomials have real coefficients. The following list shows the distribution of the roots. We record the amount of roots which are integral, irrational and in $\mathbb{C} \backslash \mathbb{R}$ up to $n \leq 50$. 


\begin{tabular}{|r|r|r|r||r|r|r|r|}
\hline$n$ & $\mathbb{Z}$ & $\mathbb{R} \backslash \mathbb{Z}$ & $\mathbb{C} \backslash \mathbb{R}$ & $n$ & $\mathbb{Z}$ & $\mathbb{R} \backslash \mathbb{Z}$ & $\mathbb{C} \backslash \mathbb{R}$ \\
\hline 1 & 1 & 0 & 0 & 26 & 4 & 16 & 6 \\
2 & 2 & 0 & 0 & 27 & 5 & 22 & 0 \\
3 & 3 & 0 & 0 & 28 & 4 & 20 & 4 \\
4 & 4 & 0 & 0 & 29 & 5 & 22 & 2 \\
5 & 3 & 2 & 0 & 30 & 3 & 19 & 8 \\
6 & 3 & 3 & 0 & 31 & 6 & 21 & 4 \\
7 & 4 & 3 & 0 & 32 & 6 & 24 & 2 \\
8 & 4 & 4 & 0 & 33 & 4 & 29 & 0 \\
9 & 6 & 3 & 0 & 34 & 6 & 18 & 10 \\
10 & 2 & 6 & 2 & 35 & 4 & 21 & 10 \\
11 & 5 & 6 & 0 & 36 & 3 & 23 & 10 \\
12 & 3 & 7 & 2 & 37 & 5 & 30 & 2 \\
13 & 5 & 8 & 0 & 38 & 4 & 28 & 6 \\
14 & 5 & 7 & 2 & 39 & 7 & 28 & 4 \\
15 & 3 & 10 & 2 & 40 & 3 & 29 & 8 \\
16 & 3 & 13 & 0 & 41 & 6 & 31 & 4 \\
17 & 6 & 11 & 0 & 42 & 6 & 30 & 6 \\
18 & 5 & 11 & 2 & 43 & 6 & 33 & 4 \\
19 & 7 & 12 & 0 & 44 & 6 & 34 & 4 \\
20 & 4 & 12 & 4 & 45 & 3 & 28 & 14 \\
21 & 3 & 14 & 4 & 46 & 4 & 30 & 12 \\
22 & 3 & 15 & 4 & 47 & 6 & 37 & 4 \\
23 & 5 & 18 & 0 & 48 & 6 & 36 & 6 \\
24 & 5 & 15 & 4 & 49 & 6 & 33 & 10 \\
25 & 4 & 17 & 4 & 50 & 4 & 38 & 8 \\
\hline
\end{tabular}

Remark. For $34 \leq n \leq 700$, we have checked numerically that $p_{n}(x)$ has always roots in $\mathbb{C} \backslash \mathbb{R}$. 
Having the vanishing property of Fourier coefficients attached to powers of the Dedekind eta function in mind, it is natural to study the integral roots of $p_{n}(x)$ in more detail. In the following we list the distribution of the possible integral roots

$$
-1,-2,-3,-4,-6,-8,-10,-14,-26 \text {. }
$$

This is done for $n \leq 50$. Note that these are all possible integral roots for this bound.

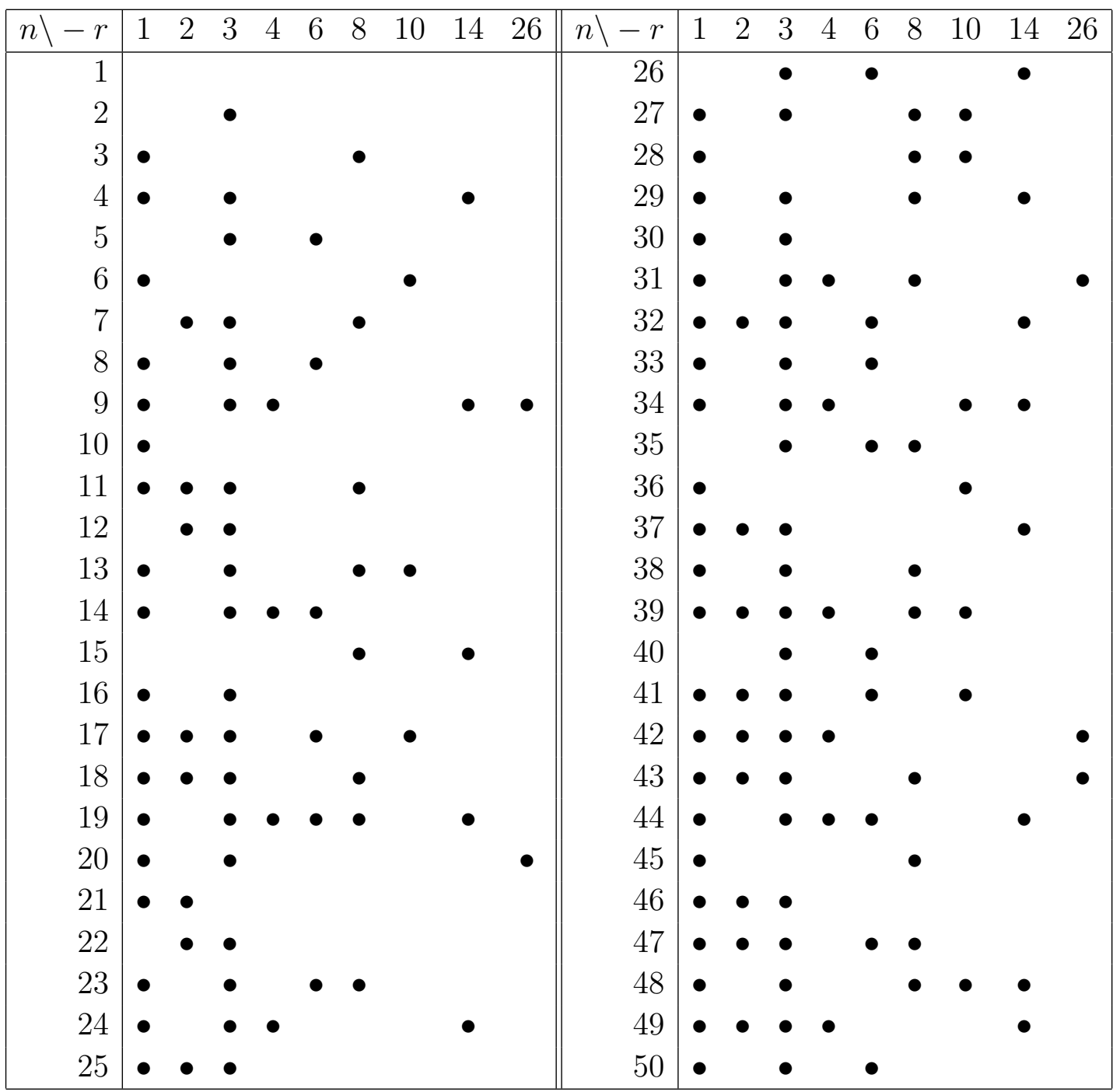

4. VArying the Imaginary Part of $p_{n}(x+i t)$

Theorem 4.1. Let $n \in \mathbb{N}$. Let $z_{0}=0, z_{1}, \ldots, z_{n-1}$ denote the roots of $p_{n}(x)$.

$$
p_{n}(x)=\frac{1}{n !} \prod_{k=0}^{n-1}\left(x-z_{k}\right) \text {. }
$$

Let $s$ be any non-negative real number. Suppose that for all $k=0, \ldots, n-1$ :

$$
\left|s-\operatorname{Re}\left(z_{k}\right)\right| \geq\left|\operatorname{Im}\left(z_{k}\right)\right|
$$


Then the function $\varphi_{s}: \mathbb{R}_{>0} \longrightarrow \mathbb{R}_{\geq 0}$,

$$
t \mapsto\left|p_{n}(s+i t)\right|
$$

is strictly increasing.

Remark. For real roots $z_{k}$, the inequality (4.1) is always satisfied.

Corollary 4.2. Let $s>0$ be fixed that satisfies the assumptions of the theorem. Then

$$
p_{n}(s+i t) \neq 0 \quad \text { for all } t \in \mathbb{R} .
$$

Proof. We know that $p_{n}(s)>0$ for $s>0$. Since

$$
p_{n}(s-i t)=\overline{p_{n}(s+i t)}
$$

this also means that $t \mapsto\left|p_{n}(s+i t)\right|$ is decreasing for $t<0$. This means that for such an $s$ the function $t \mapsto p_{n}(s+i t)$ has its minimal value at $s=0$. Hence $p_{n}(s+i t)$ cannot vanish for any $t \in \mathbb{R}$.

Proof of Theorem. We prove the theorem by studying the derivative of $\left|p_{n}(z)\right|^{2}$ with respect to the imaginary variable. Let $s=\operatorname{Re}(z)$ and $t=\operatorname{Im}(z)$. We will show that $t \mapsto\left|p_{n}(s+i t)\right|^{2}$ is increasing for $t>0$. We have

$$
\frac{d}{d t}\left|p_{n}(z)\right|^{2}=\left|p_{n}(z)\right|^{2} \sum_{k=0}^{n-1} \frac{2\left(t-\beta_{k}\right)}{\left(s-\alpha_{k}\right)^{2}+\left(t-\beta_{k}\right)^{2}} .
$$

If $\beta_{k} \neq 0$ the real polynomial also has the root $\alpha_{k}-i \beta_{k}$ different from $\alpha_{k}+i \beta_{k}$. In this case we obtain

$$
\begin{aligned}
& \frac{2\left(t-\beta_{k}\right)}{\left(s-\alpha_{k}\right)^{2}+\left(t-\beta_{k}\right)^{2}}+\frac{2\left(t+\beta_{k}\right)}{\left(s-\alpha_{k}\right)^{2}+\left(t+\beta_{k}\right)^{2}} \\
= & \frac{2\left(t-\beta_{k}\right)\left(\left(s-\alpha_{k}\right)^{2}+\left(t+\beta_{k}\right)^{2}\right)+2\left(t+\beta_{k}\right)\left(\left(s-\alpha_{k}\right)^{2}+\left(t-\beta_{k}\right)^{2}\right)}{\left(\left(s-\alpha_{k}\right)^{2}+\left(t-\beta_{k}\right)^{2}\right)\left(\left(s-\alpha_{k}\right)^{2}+\left(t+\beta_{k}\right)^{2}\right)} \\
= & \frac{2 t\left(2\left(s-\alpha_{k}\right)^{2}+\left(t+\beta_{k}\right)^{2}+\left(t-\beta_{k}\right)^{2}\right)-2 \beta_{k}\left(\left(t+\beta_{k}\right)^{2}-\left(t-\beta_{k}\right)^{2}\right)}{\left(\left(s-\alpha_{k}\right)^{2}+\left(t-\beta_{k}\right)^{2}\right)\left(\left(s-\alpha_{k}\right)^{2}+\left(t+\beta_{k}\right)^{2}\right)} \\
= & \frac{2 t\left(2\left(s-\alpha_{k}\right)^{2}+2 t^{2}+2 \beta_{k}^{2}\right)-8 t \beta_{k}^{2}}{\left(\left(s-\alpha_{k}\right)^{2}+\left(t-\beta_{k}\right)^{2}\right)\left(\left(s-\alpha_{k}\right)^{2}+\left(t+\beta_{k}\right)^{2}\right)} \\
= & \frac{2 t\left(2\left(s-\alpha_{k}\right)^{2}+2 t^{2}-2 \beta_{k}^{2}\right)}{\left(\left(s-\alpha_{k}\right)^{2}+\left(t-\beta_{k}\right)^{2}\right)\left(\left(s-\beta_{k}\right)^{2}+\left(t+\beta_{k}\right)^{2}\right)} .
\end{aligned}
$$

The last computation also holds for $\beta_{k}=0$. This last expression is $\geq 0$ if $\left|s-\alpha_{k}\right| \geq$ $\left|\beta_{k}\right|$. Hence $t \mapsto\left|p_{n}(s+i t)\right|^{2}$ is increasing if $\left|s-\alpha_{k}\right| \geq\left|\beta_{k}\right|$ for all $k$.

Corollary 4.3. $t \mapsto \mid p_{n}($ it $) \mid$ is increasing if $\left|\alpha_{k}\right| \geq\left|\beta_{k}\right|$ for all $k$.

We made the following observation for $n \leq 540$, which is maybe just a coincidence. Let

$$
S:=\{120,168,180,240,252,300,336,360,420,432,480,504,540\},
$$


then the condition of the corollary is satisfied if $n$ is not in $S$. For example the root of $p_{120}$ which violates the condition is

$$
-0.027 \ldots \pm 0.030 \ldots i \text {. }
$$

Note, that the sequence induced by $S$ does almost coincide with the numbers $n$ such that

$$
\sigma_{2}(n) / n^{2} \geq \frac{3}{2}
$$

Here $\sigma_{2}(n)=\sum_{d \mid n} d^{2}$. This sequence contains 60 and but does not contain 432 .

\section{VAlues OF $p_{n}(i t)$ FOR $t=1$ AND $t=1 / 10$}

Recall, we have already checked numerically, that for $n \leq 700$ all polynomials $p_{n}(x)$ are Hurwitz. All complex roots have negative real part. To understand the general situation we illustrate what is happening if the real part is root and $t=i$ and $i / 10$. In both cases similar patterns occur. There are sign changes of the real and imaginary part of the values $p_{n}(i t)$. Further the absolute values tend to go to infinity. We display the results of $p_{n}(i t)$ in full detail.

5.1. Value distribution of $p_{n}(i)$. In this subsection we study a special arithmetic function $F$ given by the values of $p_{n}(i)$. We consider

$$
\prod_{n=1}^{\infty}\left(1-q^{n}\right)^{-i}=\sum_{n=0}^{\infty} p_{n}(i) q^{n}
$$

and define

$$
F: \begin{cases}\mathbb{N}_{0} & \longrightarrow \mathbb{C} \\ n & \mapsto F(n):=p_{n}(i) .\end{cases}
$$

We are interested in the vanishing properties of $F$ and the value distributions. First values are given by

$$
\begin{aligned}
& F(0)=1, \quad F(1)=i, \quad F(2)=-\frac{1}{2}+\frac{3}{2} i, \\
& F(3)=-\frac{3}{2}+\frac{7}{6} i, \quad F(4)=-\frac{29}{12}+i, \quad F(5)=-\frac{7}{2}-\frac{7}{12} i .
\end{aligned}
$$

At a first glance we see sign changes in the real and imaginary part of the values. More general for $n \leq 1000$ and $\operatorname{Re}(F(n))$ and $\operatorname{Im}(F(n))$ see figure 1 and 2. See also figure 3 and 4 for $n \leq 10$.

The figures for the absolute value of $F$ for $n \leq 1000$ and $n \leq 10$ suggest that

- $\lim _{n \mapsto \infty}|F(n)|=\infty$,

- $F(n) \neq 0$ for all $n \in \mathbb{N}$. 

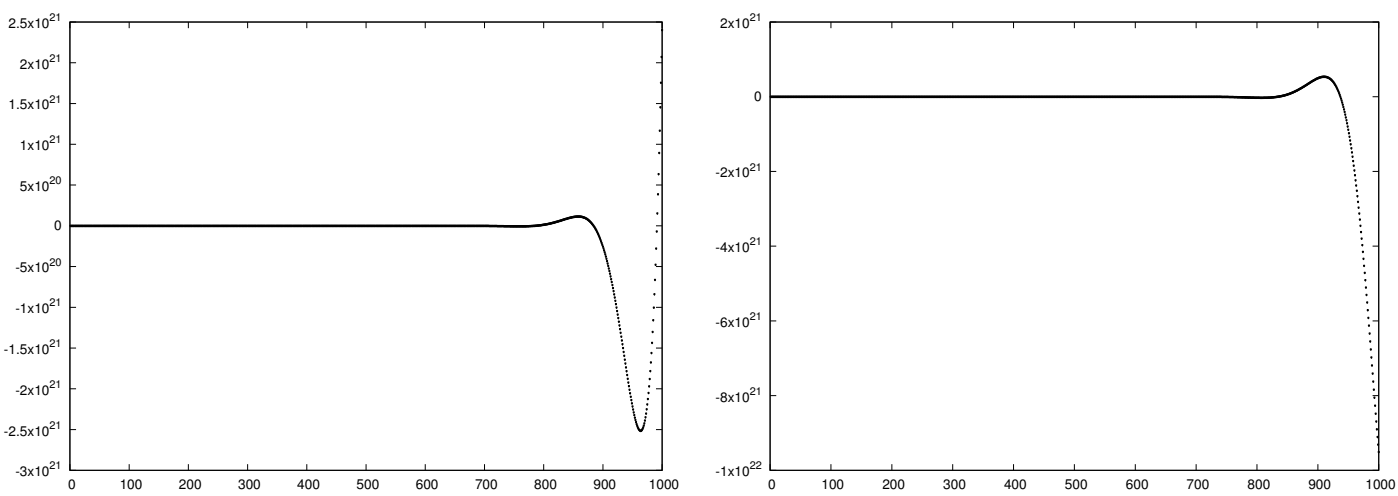

FIGURE 1. Real and imaginary parts of the sequence of the $p_{n}(i)$ for $n=1, \ldots, 1000$.
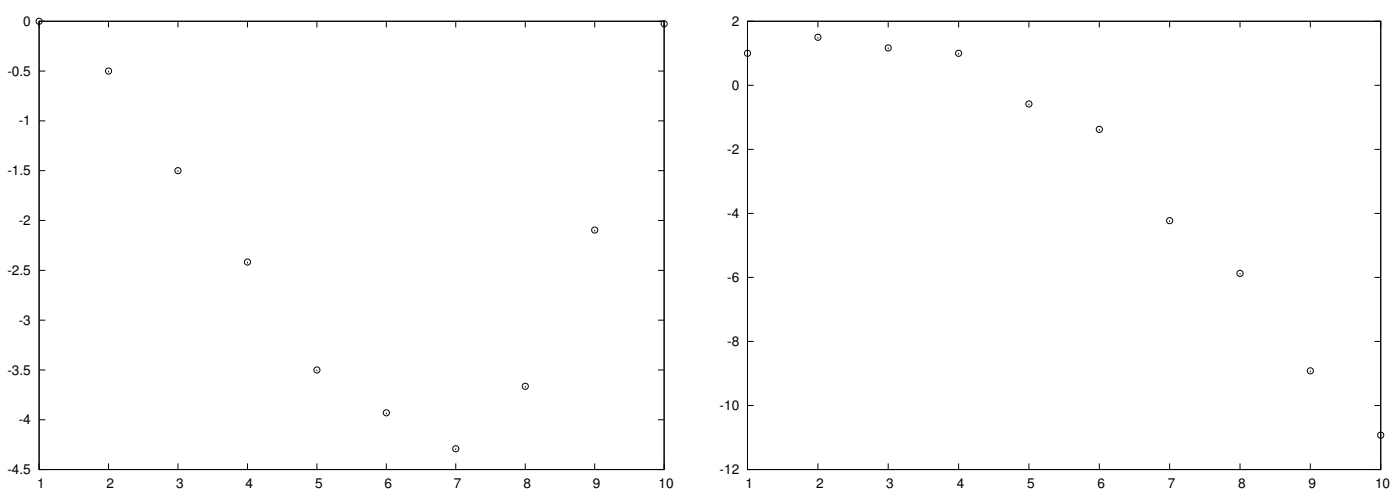

FIgURE 2. Real and imaginary parts of the sequence of the $p_{n}(i)$ for $n=1, \ldots, 10$. 


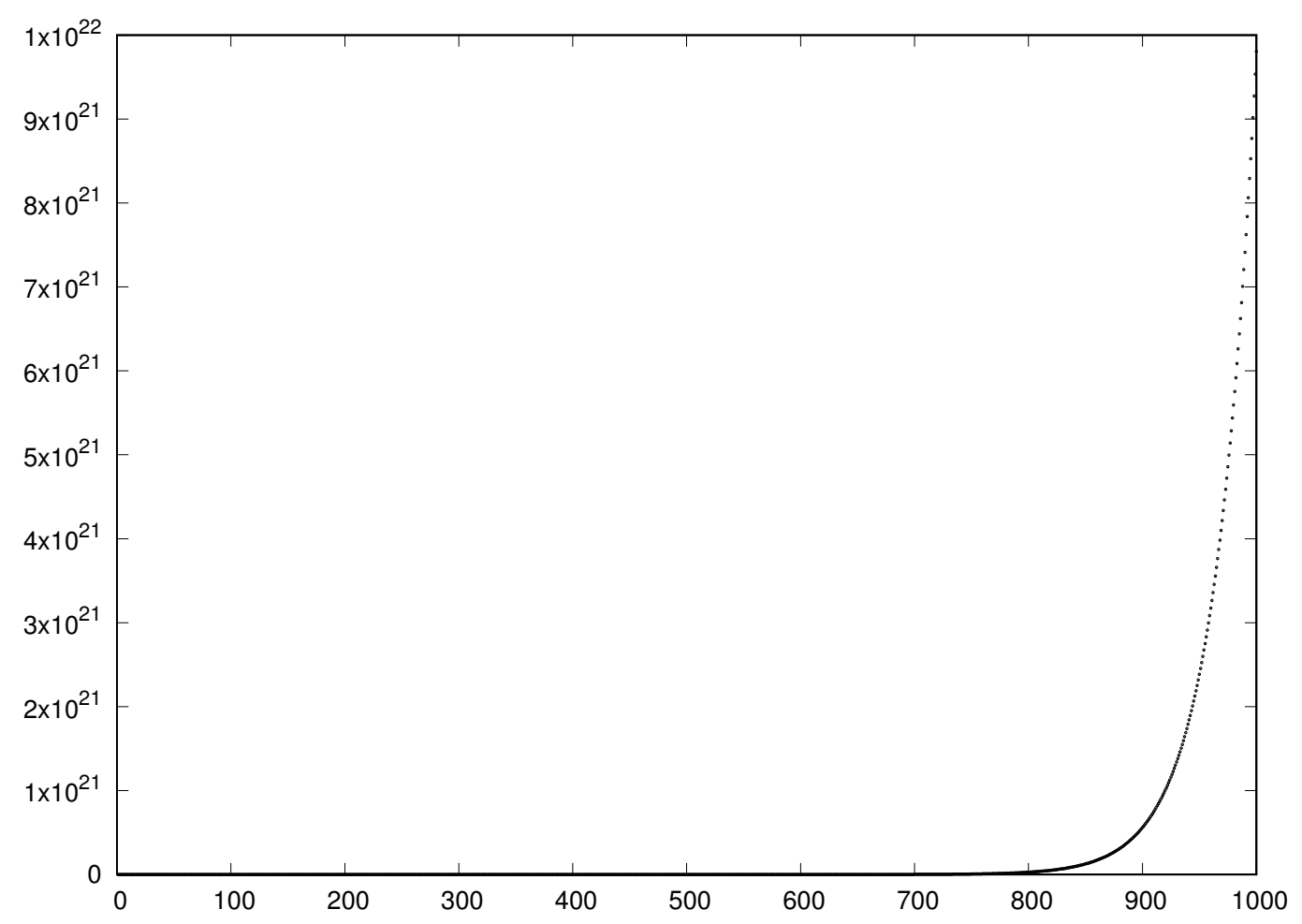

Figure 3. Absolute value of the sequence of the $p_{n}(i)$ for $n=1, \ldots, 1000$.

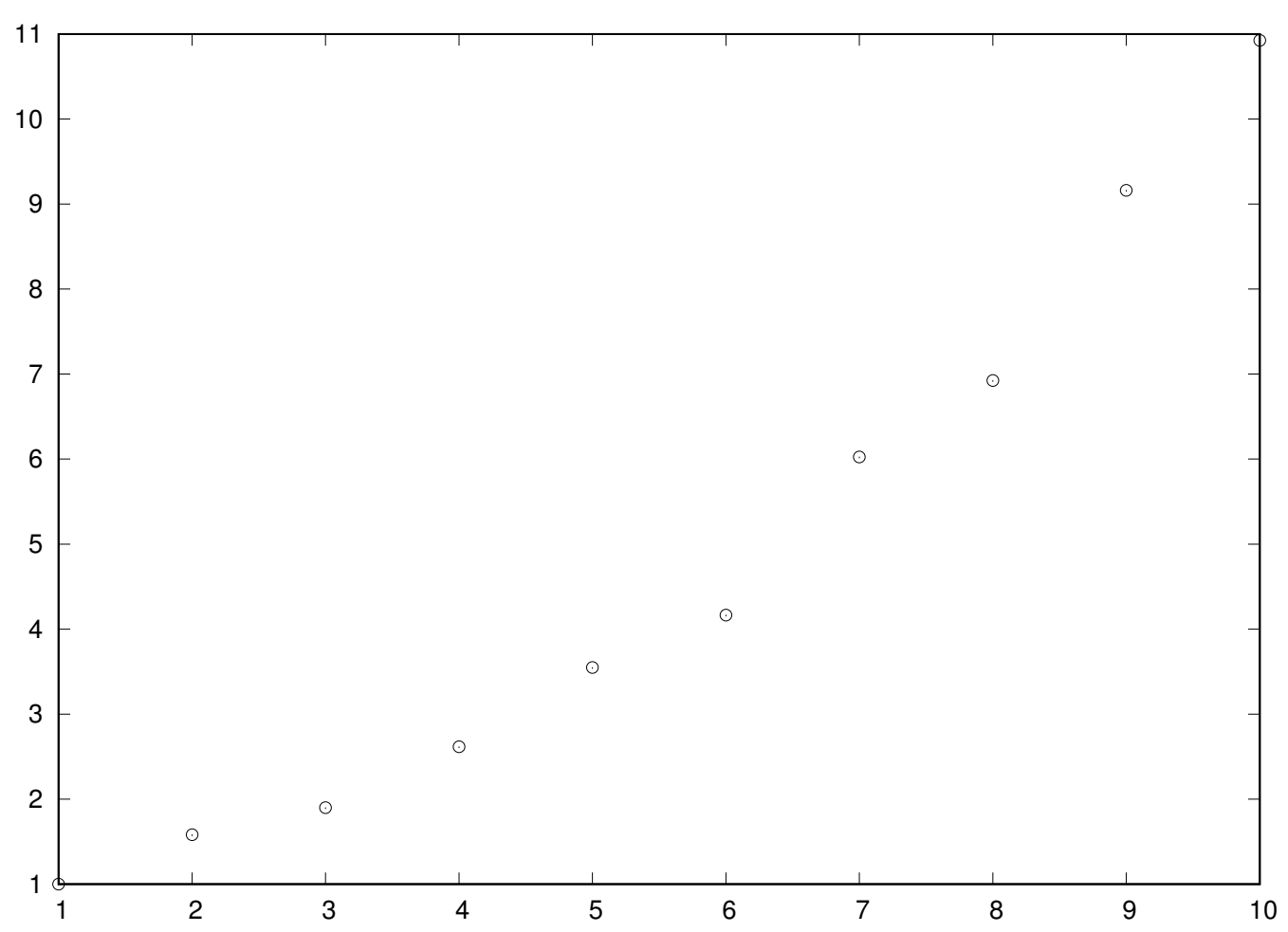

Figure 4. Absolute value of the sequence of the $p_{n}(i)$ for $n=1, \ldots, 10$. 


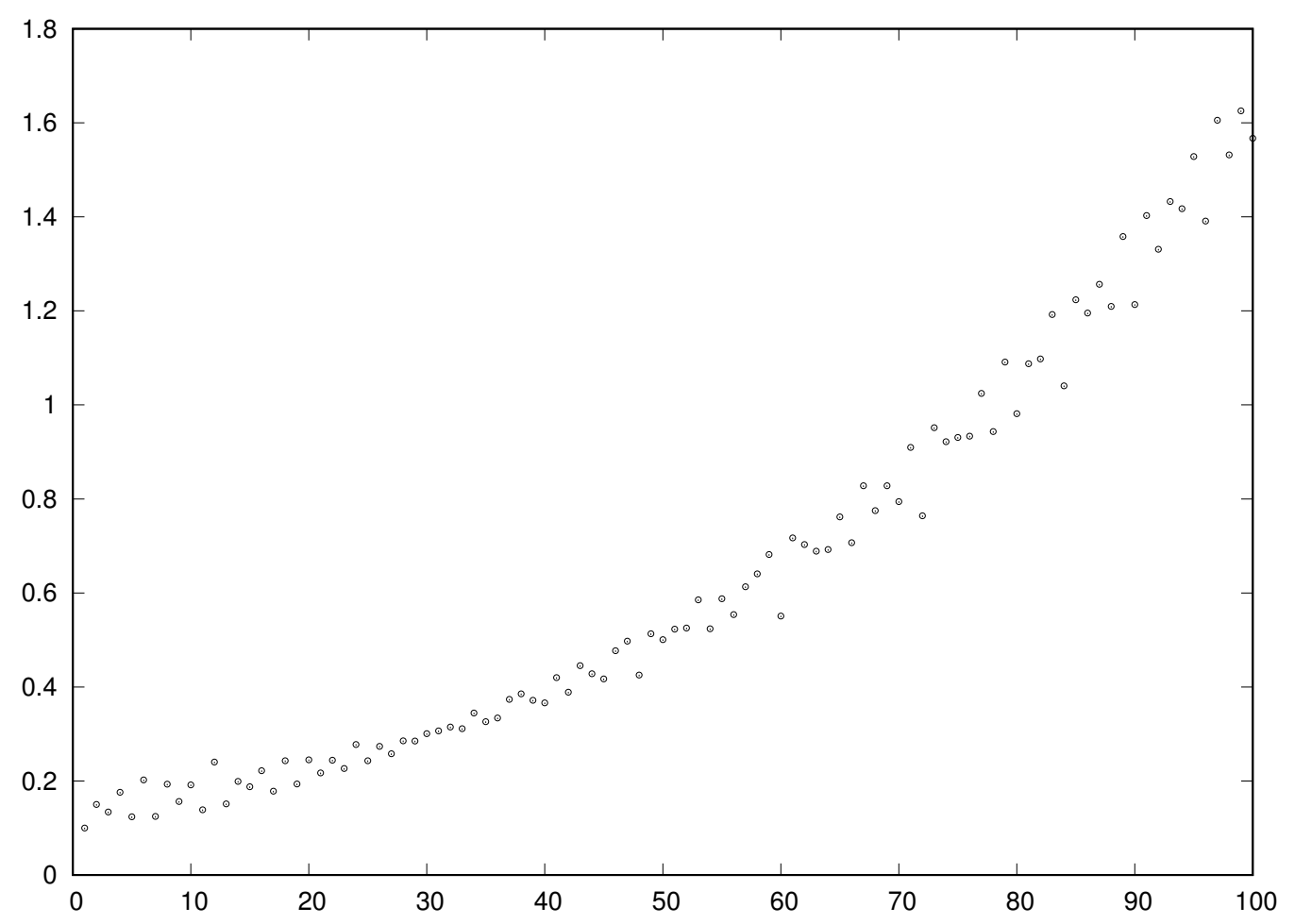

Figure 5. Absolute value of the sequence of the $p_{n}(i / 10)$ for $n=1, \ldots, 100$.

5.2. Value distribution of $p_{n}(i / 10)$. Additionally to $t=1$ we have also displayed the value distribution for $t=1 / 10$ in Figure 5 . We note that it seems that the limit of the absolute values goes to infinity. But the values are not strictly increasing. 


\section{HuRWITZ CRITERION}

Let $P(x)=\sum_{k=0}^{n} a_{n} x^{n}=a_{n} x^{n}+a_{n-1} x^{n-1}+\ldots+a_{0}$ be a real polynomial i. e. all $a_{k} \in \mathbb{R}$. It is called Hurwitz polynomial if all its roots have negative real part. Hurwitz [Hu95] showed that a polynomial has this property if and only if for the following matrix

$$
H=\left(\begin{array}{ccccc}
a_{n-1} & a_{n-3} & a_{n-5} & a_{n-7} & \cdots \\
a_{n} & a_{n-2} & a_{n-4} & a_{n-6} & \cdots \\
0 & a_{n-1} & a_{n-3} & a_{n-5} & \ldots \\
0 & a_{n} & a_{n-2} & a_{n-4} & \ldots \\
\vdots & \vdots & \vdots & \vdots & \ddots
\end{array}\right)
$$

all leading principal minors are positive.

We apply this now to the polynomials $p_{n}^{*}, n-1=1,2,3,4,5$.

We have $p_{2}^{*}(x)=x+3$ and $p_{3}^{*}(x)=x^{2}+9 x+8$. So for $n=3$ we have

$$
H_{3}=\left(\begin{array}{ll}
9 & 0 \\
1 & 8
\end{array}\right)
$$

with $9>0$ and $\operatorname{det}\left(H_{3}\right)=72>0$.

For $n=4$ we have $p_{4}^{*}(x)=z^{3}+18 z^{2}+59 z+42$ which yields

$$
H_{4}=\left(\begin{array}{ccc}
18 & 42 & 0 \\
1 & 59 & 0 \\
0 & 18 & 42
\end{array}\right)
$$

with $18>0, \operatorname{det}\left(\begin{array}{cc}18 & 42 \\ 1 & 59\end{array}\right)=1020>0$, and $\operatorname{det}\left(H_{4}\right)=1020 \cdot 42>0$.

For $n=5$ we have $p_{5}^{*}(x)=x^{4}+30 x^{3}+215 x^{2}+450 x+144$ and

$$
H_{5}=\left(\begin{array}{cccc}
30 & 450 & 0 & 0 \\
1 & 215 & 144 & 0 \\
0 & 30 & 450 & 0 \\
0 & 1 & 215 & 144
\end{array}\right)
$$

with $30>0, \operatorname{det}\left(\begin{array}{cc}30 & 450 \\ 1 & 215\end{array}\right)=6000>0$,

$$
\operatorname{det}\left(\begin{array}{ccc}
30 & 450 & 0 \\
1 & 215 & 144 \\
0 & 30 & 450
\end{array}\right)=2570400>0
$$

and $\operatorname{det}\left(H_{5}\right)=2570400 \cdot 144>0$.

(6) $p_{6}(x)=x^{5}+45 x^{4}+565 x^{3}+2475 x^{2}+3394 x+1440$, 
(i) $H_{6}=\left(\begin{array}{ccccc}45 & 2475 & 1440 & 0 & 0 \\ 1 & 565 & 3394 & 0 & 0 \\ 0 & 45 & 2475 & 1440 & 0 \\ 0 & 1 & 565 & 3394 & 0 \\ 0 & 0 & 45 & 2475 & 1440\end{array}\right)$,

(ii) $45>0, \operatorname{det}\left(\begin{array}{cc}45 & 2475 \\ 1 & 565\end{array}\right)=22950>0$,

(iii) $\operatorname{det}\left(\begin{array}{ccc}45 & 2475 & 1440 \\ 1 & 565 & 3394 \\ 0 & 45 & 2475\end{array}\right)=49993200>0$, and

(iv) $\operatorname{det}\left(\begin{array}{cccc}45 & 2475 & 1440 & 0 \\ 1 & 565 & 3394 & 0 \\ 0 & 45 & 2475 & 1440 \\ 0 & 1 & 565 & 3394\end{array}\right)=151222658400>0$.

(v) The last implies $\operatorname{det}\left(H_{6}\right)>0$.

Appendix A. The polynomials $p_{n}^{*}(x) ; n=1, \ldots, 25$

We list the polynomials $p_{n}^{*}(x)$ for $1 \leq n \leq 20$ with their factorization over $\mathbb{Q}$.

$$
\begin{aligned}
& p_{1}^{*}(x)=1 \\
& p_{2}^{*}(x)=3+x \\
& p_{3}^{*}(x)=x^{2}+9 x+8=(x+8)(x+1) \\
& p_{4}^{*}(x)=x^{3}+18 x^{2}+59 x+42=(x+14)(3+x)(x+1) \\
& p_{5}^{*}(x)=x^{4}+30 x^{3}+215 x^{2}+450 x+144=(3+x)(x+6)\left(x^{2}+21 x+8\right) \\
& p_{6}^{*}(x)=x^{5}+45 x^{4}+565 x^{3}+2475 x^{2}+3394 x+1440 \\
& =(x+10)(x+1)\left(x^{3}+34 x^{2}+181 x+144\right) \\
& p_{7}^{*}(x)=x^{6}+63 x^{5}+1225 x^{4}+9345 x^{3}+28294 x^{2}+30912 x+5760 \\
& =(x+8)(3+x)(x+2)\left(x^{3}+50 x^{2}+529 x+120\right) \\
& p_{8}^{*}(x)=x^{7}+84 x^{6}+2338 x^{5}+27720 x^{4}+147889 x^{3}+340116 x^{2}+293292 x+75600 \\
& =(x+6)(3+x)(x+1)\left(x^{4}+74 x^{3}+1571 x^{2}+9994 x+4200\right) \\
& p_{9}^{*}(x)=x^{8}+108 x^{7}+4074 x^{6}+69552 x^{5}+579369 x^{4}+2341332 x^{3}+4335596 x^{2} \\
& +3032208 x+524160 \\
& =(x+14)(x+26)(x+4)(3+x)(x+1)\left(x^{3}+60 x^{2}+491 x+120\right) \\
& p_{10}^{*}(x)=x^{9}+135 x^{8}+6630 x^{7}+154350 x^{6}+1857513 x^{5}+11744775 x^{4} \\
& +38049920 x^{3}+57773700 x^{2}+36290736 x+6531840 \\
& =(x+1)\left(x^{8}+134 x^{7}+6496 x^{6}+147854 x^{5}+1709659 x^{4}+10035116 x^{3}\right. \\
& \left.+28014804 x^{2}+29758896 x+6531840\right) \\
& p_{11}^{*}(x)=x^{10}+165 x^{9}+10230 x^{8}+311850 x^{7}+5133513 x^{6}+47002725 x^{5} \\
& +237810320 x^{4}+636593100 x^{3}+831170736 x^{2}+433762560 x+43545600 \\
& =(x+8)(3+x)(x+2)(x+1)\left(x^{6}+151 x^{5}+8057 x^{4}+190049 x^{3}\right. \\
& \left.+1983222 x^{2}+7260120 x+907200\right)
\end{aligned}
$$




$$
\begin{aligned}
& p_{12}^{*}(x)=x^{11}+198 x^{10}+15125 x^{9}+585090 x^{8}+12649263 x^{7}+158764914 x^{6} \\
& +1163220575 x^{5}+4872036510 x^{4}+11101159036 x^{3}+12532005288 x^{2} \\
& +5925016800 x+1117670400 \\
& =(3+x)(x+2)\left(x^{9}+193 x^{8}+14154 x^{7}+513162 x^{6}+9998529 x^{5}\right. \\
& \left.+105693297 x^{4}+574762916 x^{3}+1364062148 x^{2}+832270800 x+186278400\right) \\
& p_{13}^{*}(x)=x^{12}+234 x^{11}+21593 x^{10}+1033890 x^{9}+28445703 x^{8}+469839942 x^{7} \\
& +4722571139 x^{6}+28660164390 x^{5}+102004870396 x^{4}+201684764424 x^{3} \\
& +201002619168 x^{2}+83648747520 x+6706022400 \\
& =(x+10)(x+8)(3+x)(x+1) x^{8}+212 x^{7}+16774 x^{6}+631628 x^{5} \\
& \left.+11870389 x^{4}+104464688 x^{3}+344183076 x^{2}+304993872 x+27941760\right) \\
& p_{14}^{*}(x)=x^{13}+273 x^{12}+29939 x^{11}+1738737 x^{10}+59372313 x^{9}+1250368119 x^{8} \\
& +16556785817 x^{7}+137914387611 x^{6}+711131447026 x^{5}+2194635751308 x^{4} \\
& +3821401891944 x^{3}+3401283910752 x^{2}+1335385128960 x+149448499200 \\
& =(x+6)(x+4)(3+x)(x+1)\left(x^{9}+259 x^{8}+26246 x^{7}+1353814 x^{6}\right. \\
& +38627729 x^{5}+615548731 x^{4}+5178575464 x^{3}+19207997676 x^{2} \\
& +14914586880 x+2075673600) \\
& p_{15}^{*}(x)=x^{14}+315 x^{13}+40495 x^{12}+2805075 x^{11}+116473357 x^{10}+3049771725 x^{9} \\
& +51550612685 x^{8}+566030289825 x^{7}+4006881036158 x^{6} \\
& +17905744436580 x^{5}+48648442726520 x^{4}+75623303901600 x^{3} \\
& +60929911689984 x^{2}+20323375994880 x+2092278988800 \\
& =(x+14)(x+8)\left(x^{12}+293 x^{11}+33937 x^{10}+2025645 x^{9}+68108223 x^{8}\right. \\
& +1324518579 x^{7}+14783082971 x^{6}+92456383615 x^{5}+317135303876 x^{4} \\
& \left.+573652786428 x^{3}+508927390992 x^{2}+177789219840 x+18681062400\right) \\
& p_{16}^{*}(x)=x^{15}+360 x^{14}+53620 x^{13}+4368000 x^{12}+216834982 x^{11}+6915788880 x^{10} \\
& +145516059260 x^{9}+2041046092800 x^{8}+19052174174033 x^{7} \\
& +116869372980360 x^{6}+459649616109320 x^{5}+1113697174699200 x^{4} \\
& +1560949651635984 x^{3}+1143429812726400 x^{2}+376785057196800 x \\
& +40537905408000 \\
& =(3+x)(x+1)\left(x^{13}+356 x^{12}+52193 x^{11}+4158160 x^{10}+200045763 x^{9}\right. \\
& +6103131348 x^{8}+120503396579 x^{7}+1540723112440 x^{6}+12527771534536 x^{5} \\
& +62136117504896 x^{4}+173521831486128 x^{3}+233201496240000 x^{2} \\
& +107578172217600 x+13512635136000) \\
& p_{17}^{*}(x)=x^{16}+408 x^{15}+69700 x^{14}+6597360 x^{13}+385985782 x^{12}+14741746656 x^{11} \\
& +378267538220 x^{10}+6609649984080 x^{9}+78849375466433 x^{8} \\
& +637855242288264 x^{7}+3443059993897160 x^{6}+12069423054212160 x^{5} \\
& +26348214301856784 x^{4}+33612562257132672 x^{3}+22572470529457920 x^{2} \\
& +6493118120294400 x+376610217984000 \\
& =(x+10)(x+6)(3+x)(x+2)(x+1)\left(x^{11}+386 x^{10}+61041 x^{9}+5189454 x^{8}\right. \\
& +261413979 x^{7}+8090623902 x^{6}+153758707859 x^{5}+1730192640946 x^{4} \\
& +10522817826720 x^{3}+27863238700512 x^{2}+15839546284800 x \\
& +1046139494400)
\end{aligned}
$$




$$
\begin{aligned}
& p_{18}^{*}(x)=x^{17}+459 x^{16}+89148 x^{15}+9703260 x^{14}+660951942 x^{13}+29796417378 x^{12} \\
& +916590073516 x^{11}+19547135742420 x^{10}+290643272718513 x^{9} \\
& +3005469776175867 x^{8}+21393928537769424 x^{7}+102896818979343480 x^{6} \\
& +324833515162163344 x^{5}+644649799518037296 x^{4}+753265741573302912 x^{3} \\
& +468013463441475840 x^{2}+132672192555571200 x+13871809695744000 \\
& =(x+8)(3+x)(x+2)(x+1)\left(x^{13}+445 x^{12}+82859 x^{11}+8516885 x^{10}\right. \\
& +536784993 x^{9}+21771121155 x^{8}+579319498337 x^{7}+10101300017735 x^{6} \\
& +112972971000106 x^{5}+772370434468900 x^{4}+2938007628611304 x^{3} \\
& \left.+5090534870258880 x^{2}+2198053442390400 x+288996035328000\right) \\
& p_{19}^{*}(x)=x^{18}+513 x^{17}+112404 x^{16}+13941972 x^{15}+1094076582 x^{14}+57506558886 x^{13} \\
& +2090372264188 x^{12}+53486430829884 x^{11}+971152877510673 x^{10} \\
& +12520831224647889 x^{9}+113940510141447912 x^{8}+722588147763843816 x^{7} \\
& +3128796899659725904 x^{6}+8973226283666266512 x^{5} \\
& +16307166492297962496 x^{4}+17581309343532995328 x^{3} \\
& +10124124979606179840 x^{2}+2513351450024755200 x+128047474114560000 \\
& =(x+14)(x+8)(x+6)(x+4)(3+x)(x+1)\left(x^{12}+477 x^{11}+94745 x^{10}\right. \\
& +10295685 x^{9}+675769623 x^{8}+27859750011 x^{7}+724716810995 x^{6} \\
& +11580058500615 x^{5}+105918767344876 x^{4}+473381655680412 x^{3} \\
& \left.+688599907461360 x^{2}+280768376476800 x+15878903040000\right) \\
& p_{20}^{*}(x)=x^{19}+570 x^{18}+139935 x^{17}+19622250 x^{16}+1757721426 x^{15}+106580794500 x^{14} \\
& +4521894989470 x^{13}+136830847448100 x^{12}+2982559682950341 x^{11} \\
& +46974405357323730 x^{10}+533059026033824355 x^{9}+4321657983197020050 x^{8} \\
& +24669235359489920536 x^{7}+97003598589686195760 x^{6} \\
& +254624013222895790640 x^{5}+426456104805385005600 x^{4} \\
& +426308057891200797696 x^{3}+229777212625132285440 x^{2} \\
& +56577426980420505600 x+5109094217170944000 \\
& =(x+26)(3+x)(x+1)\left(x^{16}+540 x^{15}+123628 x^{14}+15855552 x^{13}\right. \\
& +1268784550 x^{12}+66811070952 x^{11}+2380566181004 x^{10}+58166112231216 x^{9} \\
& +977644471112177 x^{8}+11235613053099996 x^{7}+86845719277786688 x^{6} \\
& +437819539434970032 x^{5}+1365779395575844272 x^{4}+2409559899201712512 x^{3} \\
& \left.+2048896844301415680 x^{2}+635497406843443200 x+65501207912448000\right)
\end{aligned}
$$




\section{REFERENCES}

[AL13] B. Aguirre-Hernandez, C. Loredo-Villalobos: Open Problems On Hurwitz Polynomials. Physcon 2013, San Luis Potosi, Mexico, 26-29th August, 2013.

[Ap76] T. Apostol: Introduction to analytic number theory. Springer, Berlin-Heidelberg-New York (1976).

[Hu95] A. Hurwitz: Bedingungen, unter welchen eine Gleichung nur Wurzeln mit negativen reellen Teilen besitzt. Mathematische Annalen 46 (1895), 273-285.

[KK07] M. Koecher, A. Krieg: Elliptische Funktionen und Modulformen. Springer, BerlinHeidelberg-New York (2007).

[Ko11] G. Köhler: Eta Products and Theta Series Identities. Springer Monographs in Mathematics, Springer, Berlin-Heidelberg-New York (2011).

[Le47] D. Lehmer: The vanishing of Ramanujan's $\tau(n)$. Duke Math. J. 14 (1947), 429-433.

[Ma68] J.C. Maxwell: On governors. Proceedings of the Royal Society.

[Ne55] M. Newman: An identity for the coefficients of certain modular forms. J. London Math. Soc., 30 (1955), 488-493.

[Ne56] M. Newman: A Table of the Coefficients of the Powers of $\eta(\tau)$. Proc. Acad. Amsterdam 59 (1956), 204-216.

[Neh85] E. Neher: Jacobis Tripleprodukt-Identität und $\eta$-Identitäten in der Theorie der affinen Lie-Algebren. Jahresbericht d. Dt. Math.-Vereinigung 87 (1985), 164-181.

[On95] K. Ono: A note on the Shimura correspondence and the Ramanujan $\tau(n)$ function. Utilitas Mathematica 47 (1995), 170-180.

[OS95] K. Ono and S. Robins: Superlacunary Cusp Forms. Proceedings of the AMS 123 N.4 (1995), 1021-1029.

[On98] K. Ono: Gordons $\epsilon$-conjecture on the lacunarity of modular forms. C. R. Math. Rep. Acad. Sci. Canada 20 (1998), 103-107.

[On03] K. Ono: The Web of Modularity: Arithmetic of the Coefficients of Modular Forms and q-series. Conference Board of Mathematical Sciences 102 (2003).

[Ri77] K. Ribet: Galois Representations attached to Eigenforms with Nebentypus. Lect. Notes in Math. 601 (1977), 17-52.

[Se81] J. Serre: Quelques applications du théorèm de densité de Chebotarov. Inst. Hautes Études Sci. Pub. Math No. 54 (1981), 323-401.

[Se85] J. Serre: Sur la lacunarité des puissances de $\eta$. Glasgow Math. J. 27 (1985), 203-221.

German University of Technology in Oman, Muscat, Sultanate of Oman

MaX-Planck-Institute for Mathematics in Bonn, 53111 Bonn, Vivatsgasse 7, GerMANY

E-mail address: bernhard.heim@gutech.edu.om, heim@mpim-bonn.mpg.de

German University of Technology in Oman, Muscat, Sultanate of Oman

Faculty of Mathematics, Computer Science, and Natural Sciences, RWTH Aachen University, 52056 Aachen, Germany

E-mail address: markus.neuhauser@gutech.edu.om, markus.neuhauser@matha.rwth-aachen.de

Center of Conflict Resolution \& Institute of SocioEconomics, Technische Universität München, Hurwitz Gesellschaft, Bolzmannstr.3,, D-85747 Garching Bei MÜNChen, GERMANY

E-mail address: rupp@ma.tum.de 